MATHEMATICS OF COMPUTATION

Volume 78, Number 268, October 2009, Pages 2209-2222

S 0025-5718(09)02217-0

Article electronically published on January 29, 2009

\title{
A NEW MULTIDIMENSIONAL CONTINUED FRACTION ALGORITHM
}

\author{
JUN-ICHI TAMURA AND SHIN-ICHI YASUTOMI
}

\begin{abstract}
It has been believed that the continued fraction expansion of $(\alpha, \beta)$ $(1, \alpha, \beta$ is a $\mathbb{Q}$-basis of a real cubic field) obtained by the modified JacobiPerron algorithm is periodic. We conducted a numerical experiment (cf. Table $\mathrm{B}$, Figure 1 and Figure 2) from which we conjecture the non-periodicity of the expansion of $(\langle\sqrt[3]{3}\rangle,\langle\sqrt[3]{9}\rangle)(\langle x\rangle$ denoting the fractional part of $x)$. We present a new algorithm which is something like the modified Jacobi-Perron algorithm, and give some experimental results with this new algorithm. From our experiments, we can expect that the expansion of $(\alpha, \beta)$ with our algorithm always becomes periodic for any real cubic field. We also consider real quartic fields.
\end{abstract}

\section{INTRODUCTION}

The study of continued fractions has a long history dating back to J. Wallis (1616-1703) and Ch. Huygens (1629-1695) [8]. In particular, many kinds of higherdimensional continued fractions have been studied starting with K.G. Jacobi (18041851) [7. A central problem has been to find a higher-dimensional generalization of Legendre's theorem concerning the periodic continued fractions. In fact, the following conjecture has been believed.

Conjecture. Let $1, \alpha_{1}, \ldots, \alpha_{s}$ be a $\mathbb{Q}$-basis of real number field $K$ with $[K: \mathbb{Q}]=$ $s+1$. Then, the expansion of $\left(\alpha_{1}, \ldots, \alpha_{s}\right)$ by the Jacobi-Perron algorithm is eventually periodic; cf. [11].

Although Bernstein [1] gave some classes of periodic continued fractions obtained by the Jacobi-Perron algorithm, the conjecture is still open. For example, the periodicity of the Jacobi-Perron algorithm for $\left(\sqrt[3]{4}, \sqrt[3]{4^{2}}\right)$ has not been established (see [2], [12]). We also gave a numerical experiments (cf. Table B, Figure 1 and Figure 2) from which we conjecture the non-periodicity of the expansion of $(\langle\sqrt[3]{3}\rangle,\langle\sqrt[3]{9}\rangle)$ by the modified Jacobi-Perron algorithm.

In this paper, we give some candidates of algorithms of continued fraction expansion of dimensions 2 and 3, which can be easily generalized to any dimension.

By numerical experiments, we checked that, for instance, $\left(\langle\sqrt[3]{m}\rangle,\left\langle\sqrt[3]{m^{2}}\right\rangle\right)(2 \leq$ $m \leq 5000, m \in \mathbb{Z}, \sqrt[3]{m} \notin \mathbb{Q})$ and $\left(\langle\sqrt[4]{m}\rangle,\left\langle\sqrt[4]{m^{2}}\right\rangle,\left\langle\sqrt[4]{m^{3}}\right\rangle\right)(2 \leq m \leq 5000, m \in$ $\mathbb{Z}, \sqrt{m} \notin \mathbb{Q})$ obtained by our algorithm become periodic. We showed the periodicity

Received by the editor May 8, 2008 and, in revised form, August 25, 2008.

2000 Mathematics Subject Classification. Primary 11J70; Secondary 68W25.

Key words and phrases. Diophantine approximation, multidimensional continued fraction algorithm.

(C)2009 American Mathematical Society Reverts to public domain 28 years from publication 
of the expansion of $(\alpha, \beta) \in K^{2}$ by our algorithm for small classes of cubic fields $K$, including totally real cases, and pure cubic cases; cf. Theorems 2.4, 2.5,

The algorithms given in this paper are motivated by the algorithms given in [12, which are quite different from the Jacobi-Perron algorithm, but our algorithms are related to the so-called modified Jacobi-Perron algorithm; cf. [3], 4, [5], 10].

\section{The CUBIC CASE}

In this section we consider real cubic fields $K$ (including totally real cases and not totally real cases). We denote by $X_{K}$ the set defined by

$X_{K}:=\left\{(\alpha, \beta) \in K^{2} \mid 1, \alpha, \beta\right.$ are linearly independent over $\left.\mathbb{Q}\right\} \cap I^{2}$, where $I=[0,1)$.

We define the transformation $T_{K}$ on $X_{K}$ by

$$
T_{K}(\alpha, \beta):= \begin{cases}\left(\frac{1}{\alpha}-\left\lfloor\frac{1}{\alpha}\right\rfloor, \frac{\beta}{\alpha}-\left\lfloor\frac{\beta}{\alpha}\right\rfloor\right) & \text { if } \frac{\alpha}{\sqrt{|N(\alpha)|}}>\frac{\beta}{\sqrt{|N(\beta)|}} \\ \left(\frac{\alpha}{\beta}-\left\lfloor\frac{\alpha}{\beta}\right\rfloor, \frac{1}{\beta}-\left\lfloor\frac{1}{\beta}\right\rfloor\right) & \text { if } \frac{\alpha}{\sqrt{|N(\alpha)|}}<\frac{\beta}{\sqrt{|N(\beta)|}}\end{cases}
$$

for $(\alpha, \beta) \in X_{K}$, where $\lfloor x\rfloor$ is the floor function of $x$ and $N(x)$ is the norm of $x \in K$ over $\mathbb{Q}$.

Lemma 2.1. The transformation $T_{K}$ is well defined.

Proof. Let $(\alpha, \beta) \in X_{K}$. It suffices to show that $\frac{\alpha}{\sqrt{|N(\alpha)|}} \neq \frac{\beta}{\sqrt{|N(\beta)|}}$. We suppose $\frac{\alpha}{\sqrt{|N(\alpha)|}}=\frac{\beta}{\sqrt{|N(\beta)|}}$. Then, we have $\alpha=\sqrt{\frac{|N(\alpha)|}{|N(\beta)|}} \beta$. Since $\alpha$ and $\beta$ are linearly independent over $\mathbb{Q}$, so that $\sqrt{\frac{|N(\alpha)|}{|N(\beta)|}} \notin \mathbb{Q}$. Hence $\sqrt{\frac{|N(\alpha)|}{|N(\beta)|}}$ is a quadratic irrational and $\sqrt{\frac{|N(\alpha)|}{|N(\beta)|}} \in K$, which is a contradiction. It is easy to see that $T_{K}(\alpha, \beta) \in$ $X_{K}$.

We define the integer-valued functions $a, b$ and $e$ on $X_{K}$ as follows:

$$
\begin{aligned}
& a(\alpha, \beta):= \begin{cases}\left\lfloor\frac{1}{\alpha}\right\rfloor & \text { if } \frac{\alpha}{\sqrt{|N(\alpha)|}}>\frac{\beta}{\sqrt{|N(\beta)|}}, \\
\left\lfloor\frac{\alpha}{\beta}\right\rfloor & \text { if } \frac{\alpha}{\sqrt{|N(\alpha)|}}<\frac{\beta}{\sqrt{|N(\beta)|}},\end{cases} \\
& b(\alpha, \beta):= \begin{cases}\left\lfloor\frac{\beta}{\alpha}\right\rfloor & \text { if } \frac{\alpha}{\sqrt{|N(\alpha)|}}>\frac{\beta}{\sqrt{|N(\beta)|}}, \\
\left\lfloor\frac{1}{\beta}\right\rfloor & \text { if } \frac{\alpha}{\sqrt{|N(\alpha)|}}<\frac{\beta}{\sqrt{|N(\beta)|}},\end{cases} \\
& e(\alpha, \beta):= \begin{cases}0 & \text { if } \frac{\alpha}{\sqrt{|N(\alpha)|}}>\frac{\beta}{\sqrt{|N(\beta)|}} \\
1 & \text { if } \frac{\alpha}{\sqrt{|N(\alpha)|}}<\frac{\beta}{\sqrt{|N(\beta)|}}\end{cases}
\end{aligned}
$$

for $(\alpha, \beta) \in X_{K}$. 
We put

$$
\begin{aligned}
\left(a_{n}, b_{n}, e_{n}\right) & =\left(a_{n}(\alpha, \beta), b_{n}(\alpha, \beta), e_{n}(\alpha, \beta)\right) \\
& :=\left(a \left(T_{K}^{n-1}(\alpha, \beta), b\left(T_{K}^{n-1}(\alpha, \beta), e\left(T_{K}^{n-1}(\alpha, \beta)\right) \quad\left(n \in \mathbb{Z}_{>0}\right),\right.\right.\right. \\
\mathcal{S}(\alpha, \beta) & :=\left\{\left(a_{n}(\alpha, \beta), b_{n}(\alpha, \beta), e_{n}(\alpha, \beta)\right)\right\}_{n=1}^{\infty} .
\end{aligned}
$$

The sequence $\mathcal{S}(\alpha, \beta)$ will be referred to as the expansion of $(\alpha, \beta) \in X_{K}$ by $T_{K}$; $T_{K}$ gives rise to a 2 -dimensional continued fraction expansion, which will be called the Algebraic Jacobi-Perron Algorithm(AJPA). Throughout our paper $\alpha_{n}, \beta_{n}$ are numbers defined by

$$
\left(\alpha_{n}, \beta_{n}\right):=T_{K}^{n}(\alpha, \beta), \quad\left(n \in \mathbb{Z}_{\geq 0}\right)
$$

Notice that

$$
\left(a_{n}, b_{n}, e_{n}\right) \in \mathbb{Z}_{\geq 0} \times \mathbb{Z}_{\geq 0} \times\{0,1\}, \quad\left(n \in \mathbb{Z}_{\geq 0}\right) .
$$

For each $\left(a^{\prime}, b^{\prime}, e^{\prime}\right) \in \mathbb{Z}_{>0} \times \mathbb{Z}_{\geq 0} \times\{0,1\}$, we put

$$
\begin{aligned}
& A_{\left(a^{\prime}, b^{\prime}, e^{\prime}\right)}:=\left\{\begin{array}{l}
\left(\begin{array}{ccc}
0 & 0 & 1 \\
0 & 1 & b^{\prime} \\
1 & 0 & a^{\prime}
\end{array}\right) \quad \text { if } e^{\prime}=0, \\
\left(\begin{array}{ccc}
1 & 0 & a^{\prime} \\
0 & 0 & 1 \\
0 & 1 & b^{\prime}
\end{array}\right) \quad \text { if } e^{\prime}=1,
\end{array}\right. \\
& M_{n}(\alpha, \beta)=\left(\begin{array}{lll}
p_{n}^{\prime \prime}(\alpha, \beta) & p_{n}^{\prime}(\alpha, \beta) & p_{n}(\alpha, \beta) \\
q_{n}^{\prime \prime}(\alpha, \beta) & q_{n}^{\prime}(\alpha, \beta) & q_{n}(\alpha, \beta) \\
r_{n}^{\prime \prime}(\alpha, \beta) & r_{n}^{\prime}(\alpha, \beta) & r_{n}(\alpha, \beta)
\end{array}\right):=A_{\left(a_{1}, b_{1}, e_{1}\right)} \cdots A_{\left(a_{n}, b_{n}, e_{n}\right)} .
\end{aligned}
$$

Definition. We denote by $\mathcal{P}_{K}$ the set

$\left\{(\alpha, \beta) \in X_{K} \mid\right.$ there exist $m, n \in \mathbb{Z}_{>0}$ such that $m \neq n$ and $\left.T_{K}^{m}(\alpha, \beta)=T_{K}^{n}(\alpha, \beta)\right\}$.

If $(\alpha, \beta) \in \mathcal{P}_{K}$, the expansion $\mathcal{S}(\alpha, \beta)$ by $T_{K}$ becomes periodic, and vice versa. In what follows we mean by "the period" the period obtained by choosing the shortest period and preperiod. For the periodic continued fraction obtained by AJPA, we have the following proposition. In a way similar to Perron [9], we can show

Proposition 2.2. Let $(\alpha, \beta) \in \mathcal{P}_{K}$. Then, there exists a constant $c(\alpha, \beta)>0$ and $\eta(\alpha, \beta)>0$ such that $\eta(\alpha, \beta) \leq \frac{3}{2}$ and

$$
\begin{gathered}
\left|\alpha-\frac{p_{n}}{r_{n}}\right| \leq \frac{c(\alpha, \beta)}{r_{n}^{\eta(\alpha, \beta)}}, \\
\left|\beta-\frac{q_{n}}{r_{n}}\right| \leq \frac{c(\alpha, \beta)}{r_{n}^{\eta(\alpha, \beta)}},
\end{gathered}
$$

holds. Furthermore, $\eta(\alpha, \beta)=\frac{3}{2}$ holds if and only if $K$ is not a totally real cubic field.

Remark 2.3 .

(1) Based on our many experiments (cf. Tables A, C, D), we can hope that

$$
X_{K}=P_{K} \text {. }
$$


(2) In view of Proposition 2.2, we see that

$$
(\alpha, \beta) \neq\left(\alpha^{\prime}, \beta^{\prime}\right) \Longleftrightarrow \mathcal{S}(\alpha, \beta) \neq \mathcal{S}\left(\alpha^{\prime}, \beta^{\prime}\right),
$$

for $(\alpha, \beta),\left(\alpha^{\prime}, \beta^{\prime}\right) \in \mathcal{P}_{K}$.

Bernstein [1] gave some classes of periodic continued fractions obtained by the Jacobi-Perron algorithm. We can also give some examples of periodic expansions obtained by the AJPA, for example:

Theorem 2.4. Let $K=\mathbb{Q}\left(\sqrt[3]{m^{3}+1}\right)$ with $m \in \mathbb{Z}_{>0}$. Let $(\alpha, \beta)=\left(\sqrt[3]{m^{3}+1}-\right.$ $\left.m, \sqrt[3]{\left(m^{3}+1\right)^{2}}-m^{2}\right)$. Then, $(\alpha, \beta) \in \mathcal{P}_{K}$ and the length of the period is 2 .

Proof. It is easy to see that $(\alpha, \beta) \in X_{K}$. We have $|N(\alpha)|=1$ and $|N(\beta)|=$ $\left(m^{3}+1\right)^{2}-m^{6}=2 m^{3}+1$.

First, we consider the case where $m \geq 2$. Since $\sqrt{2 m^{3}+1}>\sqrt[3]{m^{3}+1}+m$, we have $\frac{\alpha}{\sqrt{|N(\alpha)|}}>\frac{\beta}{\sqrt{|N(\beta)|}}$, so that $e_{1}=0$. Therefore, we get

$$
\begin{aligned}
\left(\alpha_{1}, \beta_{1}\right) & =T_{K}(\alpha, \beta)=\left(\frac{1}{\alpha}-a_{1}, \frac{\beta}{\alpha}-b_{1}\right) \\
& =\left(\sqrt[3]{\left(m^{3}+1\right)^{2}}+m \sqrt[3]{m^{3}+1}+m^{2}-3 m^{2}, \sqrt[3]{m^{3}+1}+m-2 m\right) \\
& =\left(\sqrt[3]{\left(m^{3}+1\right)^{2}}+m \sqrt[3]{m^{3}+1}-2 m^{2}, \sqrt[3]{m^{3}+1}-m\right)
\end{aligned}
$$

We see that $\left|N\left(\alpha_{1}\right)\right|=9 m^{3}+1$ and $\left|N\left(\beta_{1}\right)\right|=1$. One can see that

$$
\frac{\sqrt[3]{\left(m^{3}+1\right)^{2}}+m \sqrt[3]{m^{3}+1}-2 m^{2}}{\sqrt{9 m^{3}+1}}<\sqrt[3]{m^{3}+1}-m .
$$

Therefore, we have $e_{2}=1$. Thus, we get

$$
\begin{aligned}
\left(\alpha_{2}, \beta_{2}\right) & \left.=T_{K}\left(\alpha_{1}, \beta_{1}\right)=\left(\frac{\alpha_{1}}{\beta_{1}}-a_{2}\right), \frac{1}{\beta_{1}}-b_{2}\right) \\
& =\left(\sqrt[3]{m^{3}+1}+2 m-3 m, \sqrt[3]{\left(m^{3}+1\right)^{2}}+m \sqrt[3]{m^{3}+1}+m^{2}-3 m^{2}\right) \\
& =\left(\sqrt[3]{m^{3}+1}-m, \sqrt[3]{\left(m^{3}+1\right)^{2}}+m \sqrt[3]{m^{3}+1}-2 m^{2}\right) \\
& =\left(\beta_{1}, \alpha_{1}\right) .
\end{aligned}
$$

Therefore, we have $\left(\alpha_{2}, \beta_{2}\right)=\left(\beta_{1}, \alpha_{1}\right)$. Hence, $\left\{T_{K}^{n}(\alpha, \beta)\right\}_{n=0}^{\infty}$ is periodic with 2 as the length of its (shortest) period. Thus, we get

\begin{tabular}{l|l|l|l}
\hline$n$ & 1 & 2 & 3 \\
\hline$a_{n}$ & $3 m^{2}$ & $3 m$ & $3 m^{2}$ \\
\hline$b_{n}$ & $2 m$ & $3 m^{2}$ & $3 m$ \\
\hline$e_{n}$ & 0 & 1 & 0 \\
\hline
\end{tabular}

$$
a_{n}=a_{n-2}, b_{n}=b_{n-2} \text { and } e_{n}=e_{n-2} \text { for all } n \geq 4 .
$$

Second, we consider the case $m=1$, i.e., $\left(\alpha_{0}, \beta_{0}\right)=(\sqrt[3]{2}-1, \sqrt[3]{4}-1)$. Then, we see that

$$
\begin{aligned}
& \left(\alpha_{1}, \beta_{1}\right)=\left(\frac{1}{3}-\frac{\sqrt[3]{2}}{3}+\frac{\sqrt[3]{4}}{3},-\frac{2}{3}+\frac{2 \sqrt[3]{2}}{3}+\frac{\sqrt[3]{4}}{3}\right) \\
& \left(\alpha_{2}, \beta_{2}\right)=\left(\alpha_{0}, \beta_{0}\right)
\end{aligned}
$$

We get 


\begin{tabular}{c|c|c}
\hline$n$ & 1 & 2 \\
\hline$a_{n}$ & 0 & 2 \\
\hline$b_{n}$ & 1 & 1 \\
\hline$e_{n}$ & 1 & 0 \\
\hline
\end{tabular}

$$
a_{n}=a_{n-2}, b_{n}=b_{n-2} \text { and } e_{n}=e_{n-2} \text { for all } n \geq 3 \text {. }
$$

We put, for $\frac{p}{q}(p, q \in \mathbb{Z}$ are coprime),

$$
\operatorname{dh}\left(\frac{p}{q}\right):=\max \left\{\left\lfloor\log _{10}|p|+1\right\rfloor,\left\lfloor\log _{10}|q|+1\right\rfloor\right\}, \quad \operatorname{dh}(0):=0 .
$$

The function dh can be extended to $\mathbb{Q}[x]$ : for $g(x)=\sum_{i=0}^{n} a_{i} x^{i} \in \mathbb{Q}[x]$, put

$$
\operatorname{dh}(g):=\max _{0 \leq i \leq n}\left\{\operatorname{dh}\left(a_{i}\right)\right\}
$$

Furthermore, we put, for $(\alpha, \beta) \in K^{2}$,

$$
\overline{\operatorname{dh}}(\alpha, \beta):=\max \{\operatorname{dh}(\operatorname{mpol}(\alpha)), \operatorname{dh}(\operatorname{mpol}(\beta))\},
$$

where $\operatorname{mpol}(\gamma) \in \mathbb{Q}[x](\gamma \in K)$ is the monic minimal polynomial of $\gamma$. We put, for $n \in \mathbb{Z}_{\geq 0}$ and $(\alpha, \beta) \in X_{K}$,

$$
\begin{aligned}
& \operatorname{dh}_{\text {AJPA }}(n ; \alpha, \beta):=\overline{\operatorname{dh}}\left(\alpha_{n}, \beta_{n}\right), \\
& \operatorname{rdh}_{\text {AJPA }}(n ; \alpha, \beta):=\frac{\overline{\operatorname{dh}}\left(\alpha_{n}, \beta_{n}\right)}{\overline{\operatorname{dh}}\left(\alpha_{0}, \beta_{0}\right)},
\end{aligned}
$$

where $\left(\alpha_{n}, \beta_{n}\right):=T_{K}^{n}(\alpha, \beta)$. The function $\operatorname{dh}_{\mathrm{AJPA}}(n ; \alpha, \beta)\left(\right.$ resp., $\left.\operatorname{rdh}_{\mathrm{AJPA}}(n ; \alpha, \beta)\right)$ is referred to as the nth decimal height of $(\alpha, \beta)$ (resp., the nth relative decimal height of $(\alpha, \beta))$ with respect to the AJPA.

Let $K=Q(\sqrt[3]{m})$ with $m \in \mathbb{Z}_{>0}$ and $\sqrt[3]{m} \notin \mathbb{Q}$. We computed the length of the periods of the expansion $\mathcal{S}\left(\langle\sqrt[3]{m}\rangle,\left\langle\sqrt[3]{m^{2}}\right\rangle\right)$ for all $m$ with $2 \leq m \leq 5000$ $(\sqrt[3]{m} \notin \mathbb{Q})$ and these decimal heights; cf. Table A. For the calculation of the tables, we used a computer equipped with GiNaC [6] on GNU C++. We confirmed that $\left(\langle\sqrt[3]{m}\rangle,\left\langle\sqrt[3]{m^{2}}\right\rangle\right) \in \mathcal{P}_{K}$ for all $m$ with $2 \leq m \leq 5000(\sqrt[3]{m} \notin \mathbb{Q})$. There are no reports on the periodicity of any of these pairs of numbers obtained by the Jacobi-Perron algorithm or any modified Jacobi-Perron algorithms except for the algorithms of 12 .

In 2] Elsner and Hasse gave numerical results for 36 pairs of cubic numbers with the Jacobi-Perron algorithm. They found 14 cases of periodicity but no sign of periodicity for the other 22 cases.

In 12 the first author computed the $\overline{\operatorname{dh}}\left(T^{n}\left(\langle\sqrt[3]{m}\rangle,\langle\sqrt[3]{m}\rangle^{2}\right)\right.$ for some $n$ and $m$, where $T$ is the transformation related to the Jacobi-Perron algorithm. Tamura observed that it becomes gradually bigger and bigger as a function of $n$ for many $2 \leq m \leq 104$. This suggests that for some $m$,

$$
\lim _{n \rightarrow \infty} \overline{\operatorname{dh}}\left(T^{n}\left(\langle\sqrt[3]{m}\rangle,\langle\sqrt[3]{m}\rangle^{2}\right)=\infty\right.
$$

i.e., an explosion of the size of minimal polynomials. We computed $\overline{\mathrm{dh}}\left(\bar{T}^{n}(\sqrt[3]{3}-\right.$ $1, \sqrt[3]{9}-2$ ) for $0 \leq n \leq 2 \times 10^{4}$, where the transformation $\bar{T}$, which is associated with the modified Jacobi-Perron algorithm, is defined by the following: 
For $(x, y) \in[0,1)^{2}(1, x, y$ are linearly independent over $\mathbb{Q})$,

$$
\bar{T}(x, y):= \begin{cases}\left(\frac{y}{x}, \frac{1}{x}-\left\lfloor\frac{1}{x}\right\rfloor\right) & \text { if } x>y, \\ \left(\frac{1}{y}-\left\lfloor\frac{1}{y}\right\rfloor, \frac{x}{y}\right) & \text { if } x<y ;\end{cases}
$$

cf. Podsypanin [10]. This algorithm has been studied in connection with simultaneous diophantine approximation 3 , 4 and [5].

Table B gives $\overline{\operatorname{dh}}\left(\bar{T}^{n}(\sqrt[3]{3}-1, \sqrt[3]{9}-2)\right.$ for $0 \leq n \leq 2 \times 10^{4}$. The table also suggests an explosion phenomenon related to the modified Jacobi-Perron algorithm.

Theorem 2.5. Let $\delta_{m}$ be the root of $x^{3}-m x+1=0(m \in \mathbb{Z}, m \geq 3)$ determined by $0<\delta_{m}<1$. Then, $K=\mathbb{Q}\left(\delta_{m}\right)$ is a cubic number field and $\left(\delta_{m}, \delta_{m}^{2}\right) \in \mathcal{P}_{K}$.

Proof. The irreduciblity of $g:=x^{3}-m x+1$ is clear. It is also clear that $g$ has a unique root $\delta_{m}$ in $[0,1)$. We consider $T_{K}^{n}(\alpha, \beta)$ with $(\alpha, \beta)=\left(\delta_{m}, \delta_{m}^{2}\right)$.

Since $\left|N\left(\delta_{m}\right)\right|=\left|N\left(\delta_{m}^{2}\right)\right|=1$ and $0<\delta_{m}<1$, we have $\frac{\delta_{m}}{\sqrt{\left|N\left(\delta_{m}\right)\right|}}>\frac{\delta_{m}^{2}}{\sqrt{\left|N\left(\delta_{m}^{2}\right)\right|}}$, so that $e_{1}=0$, and

$$
\begin{aligned}
\left(\alpha_{1}, \beta_{1}\right)=T_{K}(\alpha, \beta) & =\left(\frac{1}{\alpha}-a(\alpha, \beta), \frac{\beta}{\alpha}-b(\alpha, \beta)\right) \\
& =\left(m-\delta_{m}^{2}-(m-1), \delta_{m}\right) \\
& =\left(1-\delta_{m}^{2}, \delta_{m}\right) .
\end{aligned}
$$

Therefore,

$$
\begin{aligned}
& \frac{\alpha_{1}}{\sqrt{\left|N\left(\alpha_{1}\right)\right|}}=\frac{1-\delta_{m}^{2}}{\sqrt{\left|N\left(1-\delta_{m}^{2}\right)\right|}}=\frac{1-\delta_{m}^{2}}{\sqrt{m(m-2)}}, \\
& \frac{\beta_{1}}{\sqrt{\left|N\left(\beta_{1}\right)\right|}}=\delta_{m} .
\end{aligned}
$$

One can check that $\frac{1-\delta_{m}^{2}}{\sqrt{m(m-2)}}>\delta_{m}$ if and only if $\left(1-\delta_{m}^{2}\right)^{2}>m(m-2) \delta_{m}^{2}$. Since $\left(1-\delta_{m}^{2}\right)^{2}-m(m-2) \delta_{m}^{2}=2(m-2) \delta_{m}^{2}+2\left(\delta_{m}^{2}-\delta_{m}^{3}\right)+\delta_{m}^{4}-\delta_{m}^{6}>0$, we get $\frac{\alpha_{1}}{\sqrt{\left|N\left(\alpha_{1}\right)\right|}}>\frac{\beta_{1}}{\sqrt{\left|N\left(\beta_{1}\right)\right|}}$. Therefore, $e_{2}=0$, and

$$
\begin{aligned}
\left(\alpha_{2}, \beta_{2}\right) & =T_{K}\left(\alpha_{1}, \beta_{1}\right)=\left(\frac{1}{\alpha_{1}}-a\left(\alpha_{1}, \beta_{1}\right), \frac{\beta_{1}}{\alpha_{1}}-b\left(\alpha_{1}, \beta_{1}\right)\right) \\
& =\left(\frac{-(m-1) \delta_{m}^{2}-\delta_{m}+m^{2}-2 m+1}{m^{2}-2 m}-1, \frac{-\delta_{m}^{2}-(m-1) \delta_{m}+(m-1)}{m^{2}-2 m}\right) \\
& =\left(\frac{-(m-1) \delta_{m}^{2}-\delta_{m}+1}{m^{2}-2 m}, \frac{-\delta_{m}^{2}-(m-1) \delta_{m}+(m-1)}{m^{2}-2 m}\right) .
\end{aligned}
$$

In view of $N\left(\alpha_{2}\right)=\frac{1}{m(m-2)}$ and $N\left(\beta_{2}\right)=\frac{1}{m(m-2)}$, we get

$$
\frac{\alpha_{2}}{\sqrt{\left|N\left(\alpha_{2}\right)\right|}}=\frac{\delta^{2} \sqrt{m(m-2)}}{1-\delta_{m}^{2}}<\frac{\delta \sqrt{m(m-2)}}{1-\delta_{m}^{2}}=\frac{\beta_{2}}{\sqrt{\left|N\left(\beta_{2}\right)\right|}}
$$


Thus, we get $e_{3}=1$, and

$$
\begin{aligned}
\left(\alpha_{3}, \beta_{3}\right)=T_{K}\left(\alpha_{2}, \beta_{2}\right) & =\left(\frac{\alpha_{2}}{\beta_{2}}-a\left(\alpha_{2}, \beta_{2}\right), \frac{1}{\beta_{2}}-b\left(\alpha_{2}, \beta_{2}\right)\right) \\
& =\left(\delta_{m},-\delta_{m}^{2}-\delta_{m}+m-(m-1)\right) \\
& =\left(\delta_{m},-\delta_{m}^{2}-\delta_{m}+1\right) .
\end{aligned}
$$

Since $\left|N\left(\beta_{3}\right)\right|=m^{2}-4 m+4, \frac{\alpha_{3}}{\sqrt{\left|N\left(\alpha_{3}\right)\right|}}<\frac{\beta_{3}}{\sqrt{\left|N\left(\beta_{3}\right)\right|}}$ holds if and only if

$$
\beta_{3} \sqrt{N\left(\alpha_{3}\right)}-\alpha_{3} \sqrt{N\left(\beta_{3}\right)}=\left(-\delta_{m}^{2}-\delta_{m}+1\right)-\delta_{m}(m-2)>0,
$$

which is equivalent to

$$
-\delta_{m}^{4}+\delta_{m}^{2}-2 \delta_{m}+1>0 .
$$

The inequality (2.3) holds, since $0<\delta_{m}<\frac{1}{2}$, which can be easily seen. Therefore, we get $e_{4}=1$, and we have

$$
\begin{aligned}
\left(\alpha_{4}, \beta_{4}\right)=T_{K}\left(\alpha_{3}, \beta_{3}\right) & =\left(\frac{\alpha_{3}}{\beta_{3}}-a\left(\alpha_{3}, \beta_{3}\right), \frac{1}{\beta_{3}}-b\left(\alpha_{3}, \beta_{3}\right)\right) \\
& =\left(\frac{-\delta_{m}+1}{m-2}, \frac{-\delta_{m}^{2}+m-1}{m-2}-1\right) \\
& =\left(\frac{-\delta_{m}+1}{m-2}, \frac{-\delta_{m}^{2}+1}{m-2}\right),
\end{aligned}
$$

and $\left|N\left(\alpha_{4}\right)\right|=\frac{1}{(m-2)^{2}}$ and $\left|N\left(\beta_{4}\right)\right|=\frac{m}{(m-2)^{2}}$ follows. Thus, we have $\frac{\alpha_{4}}{\sqrt{\left|N\left(\alpha_{4}\right)\right|}}>$ $\frac{\beta_{4}}{\sqrt{\left|N\left(\beta_{4}\right)\right|}}$. Therefore, we see $e_{5}=0$ and

$$
\begin{aligned}
\left(\alpha_{5}, \beta_{5}\right)=T_{K}\left(\alpha_{4}, \beta_{4}\right) & =\left(\frac{1}{\alpha_{4}}-a\left(\alpha_{4}, \beta_{4}\right), \frac{\beta_{4}}{\alpha_{4}}-b\left(\alpha_{4}, \beta_{4}\right)\right) \\
& =\left(-\delta_{m}^{2}-\delta_{m}+m-1-(m-2), \delta_{m}+1-1\right) \\
& =\left(-\delta_{m}^{2}-\delta_{m}+1, \delta_{m}\right)
\end{aligned}
$$

which implies $\left(\alpha_{6}, \beta_{6}\right)=\left(\frac{-\delta_{m}^{2}+1}{m-2}, \frac{-\delta_{m}+1}{m-2}\right)$ and $\left(\alpha_{7}, \beta_{7}\right)=\left(\alpha_{3}, \beta_{3}\right)$. Thus, we obtain $\left(\alpha_{3+4 j}, \beta_{3+4 j}\right)=\left(\alpha_{3}, \beta_{3}\right)$ for all integer $j \geq 0$.

Thus, we get

\begin{tabular}{l|l|l|l|l|l|l|l}
\hline$n$ & 1 & 2 & 3 & 4 & 5 & 6 & 7 \\
\hline$a_{n}$ & $m-1$ & 1 & 0 & 0 & $m-2$ & 1 & 1 \\
\hline$b_{n}$ & 0 & 0 & $m-1$ & 1 & 1 & 0 & $m-2$ \\
\hline$e_{n}$ & 0 & 0 & 1 & 1 & 0 & 0 & 1 \\
\hline
\end{tabular}

$$
a_{n}=a_{n-4}, b_{n}=b_{n-4} \text { and } e_{n}=e_{n-4} \text { for all } n \geq 8 .
$$

We also confirmed that $\left(\left\langle\tau_{m}\right\rangle,\left\langle\tau_{m}^{2}\right\rangle\right) \in \mathcal{P}_{K}$ for all integers $m$ with $2 \leq m \leq 5000$, where $\tau_{m}$ is the maximal root of $x^{3}-m x+1$, while the length of the (shortest) period is very long in some cases. Table $\mathrm{C}$ gives these results. 


\section{THE QUARTIC CASE}

Let $K$ be a real quartic field over $\mathbb{Q}$. In this section, we mean by $X_{K}^{\prime}$ and $X_{K}$ the sets defined by

$$
\begin{aligned}
& X_{K}^{\prime}:=\left\{\left(\alpha_{1}, \alpha_{2}, \alpha_{3}\right) \in K^{3} \mid 1, \alpha_{1}, \alpha_{2}, \alpha_{3} \text { are linearly independent over } \mathbb{Q}\right\} \cap I^{3}, \\
& X_{K}:=\left\{\left(\alpha_{1}, \alpha_{2}, \alpha_{3}\right) \in X_{K}^{\prime} \mid \text { there exists } i \in\{1,2,3\} \text { such that } K=\mathbb{Q}\left(\alpha_{i}\right)\right\} .
\end{aligned}
$$

For $x \in K \cap I$ we define $\phi(x)$ by

$$
\phi(x):= \begin{cases}\frac{x}{\sqrt[3]{|N(x)|}} & \text { if } K=\mathbb{Q}(x), \\ -1 & \text { if } K \neq \mathbb{Q}(x) .\end{cases}
$$

In a similar manner to the cubic case, one can show the following.

Lemma 3.1. Let $\left(\alpha_{1}, \alpha_{2}, \alpha_{3}\right) \in X_{K}$. If $\phi\left(\alpha_{i}\right), \phi\left(\alpha_{j}\right)>0$ and $\phi\left(\alpha_{i}\right)=\phi\left(\alpha_{j}\right)$ for integers $i$ and $j$ with $1 \leq i, j \leq 3$, then $i=j$.

For $\alpha=\left(\alpha_{1}, \alpha_{2}, \alpha_{3}\right) \in X_{K}$, we define $\rho(\alpha)$ and

$$
\rho(\alpha)=\max \left\{\phi\left(\alpha_{i}\right) \mid i \in\{1,2,3\}\right\} .
$$

For $\alpha=\left(\alpha_{1}, \alpha_{2}, \alpha_{3}\right) \in X_{K}$, from Lemma 3.1 it follows that

$$
\sharp\left\{i \in\{1,2,3\} \mid \rho(\alpha)=\phi\left(\alpha_{i}\right)\right\}=1,
$$

and we denote by $\omega(\alpha)$ the uniquely determined number $i \in\{1,2,3\}$ with $\rho(\alpha)=$ $\phi\left(\alpha_{i}\right)$. We define the transformation $T_{K}$ on $X_{K}$ as follows: For $\alpha=\left(\alpha_{1}, \alpha_{2}, \alpha_{3}\right) \in$ $X_{K}, T_{K}(\alpha)=\left(\beta_{1}, \beta_{2}, \beta_{3}\right)$ with

$$
\beta_{i}:=\left\{\begin{array}{ll}
\frac{1}{\alpha_{i}}-\left\lfloor\frac{1}{\alpha_{i}}\right\rfloor & \text { if } i=\omega(\alpha), \\
\frac{\alpha_{i}}{\alpha_{\omega(\alpha)}}-\left\lfloor\frac{\alpha_{i}}{\alpha_{\omega(\alpha)}}\right\rfloor & \text { if } i \neq \omega(\alpha)
\end{array} \quad(i=1,2,3) .\right.
$$

We define $\mathcal{P}_{K}$, in a similar fashion in Section 2, that is,

$$
\mathcal{P}_{K}:=\left\{\alpha \in X_{K} \mid \text { there exist } m, n \in \mathbb{Z}_{>0} \text { such that } m \neq n \text { and } T_{K}^{n}(\alpha)=T_{K}^{m}(\alpha)\right\} \text {. }
$$

We also define $\overline{\mathrm{dh}}, \mathrm{dh}_{\mathrm{AJPA}}$ and $\mathrm{rdh}_{\mathrm{AJPA}}$ in a similar manner to Section 2 , namely, for $\alpha=\left(\alpha_{1}, \alpha_{2}, \alpha_{3}\right) \in X_{K}$ and $n \in \mathbb{Z}_{\geq 0}$

$$
\begin{aligned}
& \overline{\operatorname{dh}}(\alpha):=\max _{i \in\{1,2,3\}}\left\{\operatorname{dh}\left(p_{i}^{4 / \operatorname{deg}\left(p_{i}\right)}\right)\right\}, \\
& \operatorname{dh}_{\mathrm{AJPA}}(n ; \alpha):=\overline{\operatorname{dh}}\left(T_{K}^{n}(\alpha)\right), \\
& \operatorname{rdh}_{\mathrm{AJPA}}(n ; \alpha):=\frac{\overline{\operatorname{dh}}\left(T_{K}^{n}(\alpha)\right)}{\overline{\operatorname{dh}}(\alpha)},
\end{aligned}
$$

where $p_{i}=\operatorname{mpol}\left(\alpha_{i}\right) \in \mathbb{Q}[x](i \in\{1,2,3\})$ is the monic minimal polynomial of $\alpha_{i}$.

We computed the length of the period of the expansion of $\left(\langle\sqrt[4]{m}\rangle,\left\langle\sqrt[4]{m^{2}}\right\rangle,\left\langle\sqrt[4]{m^{3}}\right\rangle\right)$ obtained by $T_{K}$ for $m$ all $2 \leq m \leq 5000$ and relative decimal heights given above; cf. Table D. We confirmed that $\left(\langle\sqrt[4]{m}\rangle,\left\langle\sqrt[4]{m^{2}}\right\rangle,\left\langle\sqrt[4]{m^{3}}\right\rangle\right) \in \mathcal{P}_{K}$ for all $2 \leq m \leq$ $5000(\sqrt{m} \notin \mathbb{Q})$. 


\section{A conjecture}

Conjecture. Let $K$ be a real cubic field or a real quartic field. Then:

(1) $X_{K}=\mathcal{P}_{K}$.

(2) There exists an absolute constant $c$ independent of $K$ and $\alpha \in X_{K}$ such that $\operatorname{rdh}_{\mathrm{AJPA}}(\alpha) \leq c$ holds. (Probably we can take $c=6$.)

(3) There are infinitely many $\alpha \in X_{K}$ (including $(\langle\sqrt[3]{3}\rangle,\langle\sqrt[3]{9}\rangle)$ ) such that the expansion of $\alpha$ obtained by the Jacobi-Perron Algorithm modified by Podsypanin is not periodic.

In Table A, $L\left(m_{1}, m_{2}\right), H\left(m_{1}, m_{2}\right)$ and $R\left(m_{1}, m_{2}\right)$ are numbers defined by $L\left(m_{1}, m_{2}\right):=$ the maximum value of the length of the shortest period of the expansion of $\left(\langle\sqrt[3]{m}\rangle,\left\langle\sqrt[3]{m^{2}}\right\rangle\right)$ by our algorithm for $m_{1} \leq m \leq m_{2}$ with $\sqrt[3]{m} \notin \mathbb{Q}$,

$$
\begin{aligned}
H\left(m_{1}, m_{2}\right) & :=\max _{m_{1} \leq m \leq m_{2}, \sqrt[3]{m} \notin \mathbb{Q}, 0 \leq n<\infty} \operatorname{dh}_{\mathrm{AJPA}}\left(n ;\langle\sqrt[3]{m}\rangle,\left\langle\sqrt[3]{m^{2}}\right\rangle\right), \\
R\left(m_{1}, m_{2}\right) & :=\max _{m_{1} \leq m \leq m_{2}, \sqrt[3]{m} \notin \mathbb{Q}, 0 \leq n<\infty} \operatorname{rdh}_{\mathrm{AJPA}}\left(n ;\langle\sqrt[3]{m}\rangle,\left\langle\sqrt[3]{m^{2}}\right\rangle\right),
\end{aligned}
$$

which are well defined by the periodicity.

\section{Table A}

\begin{tabular}{l|r|r|r}
\hline $\begin{array}{l}\text { range of } m \\
m_{1} \leq m \leq m_{2}\end{array}$ & $L\left(m_{1}, m_{2}\right)$ & $H\left(m_{1}, m_{2}\right)$ & $R\left(m_{1}, m_{2}\right)$ \\
\hline $2 \leq m \leq 200$ & 494 & 5 & $3 / 2$ \\
\hline $201 \leq m \leq 400$ & 898 & 6 & $3 / 2$ \\
\hline $401 \leq m \leq 600$ & 1702 & 6 & $3 / 2$ \\
\hline $601 \leq m \leq 800$ & 1938 & 6 & $6 / 5$ \\
\hline $801 \leq m \leq 1000$ & 2802 & 7 & $7 / 5$ \\
\hline $1001 \leq m \leq 1200$ & 4062 & 7 & $7 / 5$ \\
\hline $1201 \leq m \leq 1400$ & 5586 & 7 & $7 / 5$ \\
\hline $1401 \leq m \leq 1600$ & 5090 & 8 & $8 / 5$ \\
\hline $1601 \leq m \leq 1800$ & 8022 & 7 & $7 / 5$ \\
\hline $1801 \leq m \leq 2000$ & 7854 & 8 & $8 / 5$ \\
\hline $2001 \leq m \leq 2200$ & 5486 & 7 & $7 / 5$ \\
\hline $2201 \leq m \leq 2400$ & 6422 & 7 & $7 / 5$ \\
\hline $2401 \leq m \leq 2600$ & 7758 & 8 & $7 / 5$ \\
\hline $2601 \leq m \leq 2800$ & 6026 & 8 & $4 / 3$ \\
\hline $2801 \leq m \leq 3000$ & 9970 & 8 & $4 / 3$ \\
\hline $3001 \leq m \leq 3200$ & 11562 & 8 & $4 / 3$ \\
\hline $3201 \leq m \leq 3400$ & 6734 & 9 & $3 / 2$ \\
\hline $3401 \leq m \leq 3600$ & 6650 & 8 & $4 / 3$ \\
\hline $3601 \leq m \leq 3800$ & 12350 & 8 & $4 / 3$ \\
\hline $3801 \leq m \leq 4000$ & 19230 & 8 & $4 / 3$ \\
\hline $4001 \leq m \leq 4200$ & 11454 & 8 & $4 / 3$ \\
\hline $4201 \leq m \leq 4400$ & 16410 & 8 & $4 / 3$ \\
\hline $4401 \leq m \leq 4600$ & 14618 & 8 & $4 / 3$ \\
\hline $4601 \leq m \leq 4800$ & 18158 & 8 & $4 / 3$ \\
\hline $4801 \leq m \leq 5000$ & 14918 & 8 & $4 / 3$ \\
\hline & & &
\end{tabular}


In Table B, $\underline{U}\left(m_{1}, m_{2}\right), \underline{V}\left(m_{1}, m_{2}\right)$ are numbers defined by

$$
\begin{aligned}
& \underline{U}\left(m_{1}, m_{2}\right):=\max _{m_{1} \leq n \leq m_{2}} \overline{\operatorname{dh}}\left(\bar{T}^{n}(\sqrt[3]{3}-1, \sqrt[3]{9}-2),\right. \\
& \underline{V}\left(m_{1}, m_{2}\right):=\min _{m_{1} \leq n \leq m_{2}} \overline{\operatorname{dh}}\left(\bar{T}^{n}(\sqrt[3]{3}-1, \sqrt[3]{9}-2) .\right.
\end{aligned}
$$

We define integral-valued functions $U$ and $V$ by

$$
\begin{aligned}
& U(x):=\underline{U}\left(1000 n_{1}+1,1000\left(n_{1}+1\right)\right), \text { if } 1000 n_{1}<x \leq 1000\left(n_{1}+1\right), \\
& V(x):=\underline{V}\left(1000 n_{1}+1,1000\left(n_{1}+1\right)\right), \text { if } 1000 n_{1}<x \leq 1000\left(n_{1}+1\right),
\end{aligned}
$$

for $x, n_{1} \in \mathbb{Z}_{\geq 0}$.

\section{TABLE B}

\begin{tabular}{l|r|r}
\hline range of $n$ & $\underline{U}\left(m_{1}, m_{2}\right)$ & $\underline{V}\left(m_{1}, m_{2}\right)$ \\
\hline $0 \leq n \leq 1000$ & 93 & 1 \\
\hline $1001 \leq n \leq 2000$ & 175 & 92 \\
\hline $2001 \leq n \leq 3000$ & 253 & 174 \\
\hline $3001 \leq n \leq 4000$ & 321 & 252 \\
\hline $4001 \leq n \leq 5000$ & 397 & 319 \\
\hline $5001 \leq n \leq 6000$ & 484 & 396 \\
\hline $6001 \leq n \leq 7000$ & 555 & 483 \\
\hline $7001 \leq n \leq 8000$ & 624 & 554 \\
\hline $8001 \leq n \leq 9000$ & 716 & 624 \\
\hline $9001 \leq n \leq 10000$ & 773 & 716 \\
\hline $10001 \leq n \leq 11000$ & 850 & 772 \\
\hline $11001 \leq n \leq 12000$ & 923 & 847 \\
\hline $12001 \leq n \leq 13000$ & 1000 & 921 \\
\hline $13001 \leq n \leq 14000$ & 1074 & 1000 \\
\hline $14001 \leq n \leq 15000$ & 1139 & 1073 \\
\hline $15001 \leq n \leq 16000$ & 1212 & 1137 \\
\hline $16001 \leq n \leq 17000$ & 1284 & 1211 \\
\hline $17001 \leq n \leq 18000$ & 1370 & 1284 \\
\hline $18001 \leq n \leq 19000$ & 1444 & 1370 \\
\hline $19001 \leq n \leq 20000$ & 1514 & 1444 \\
\hline
\end{tabular}




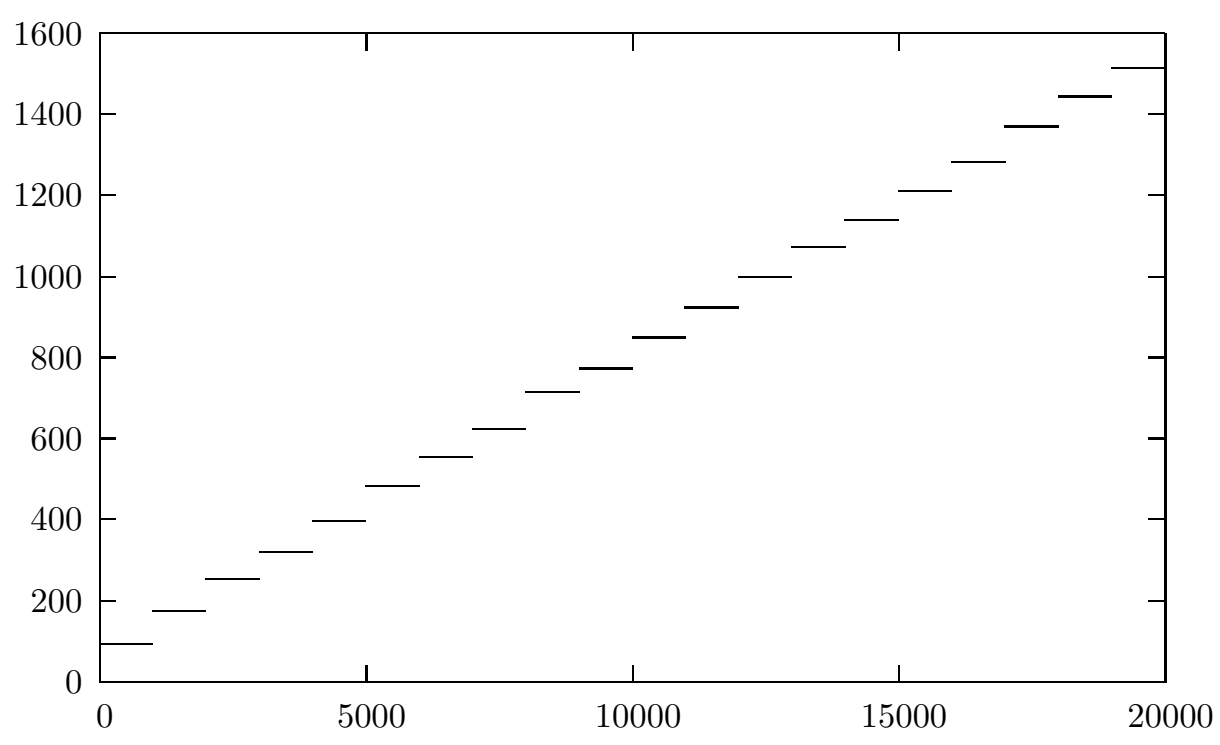

Figure 1. The graph of $U$

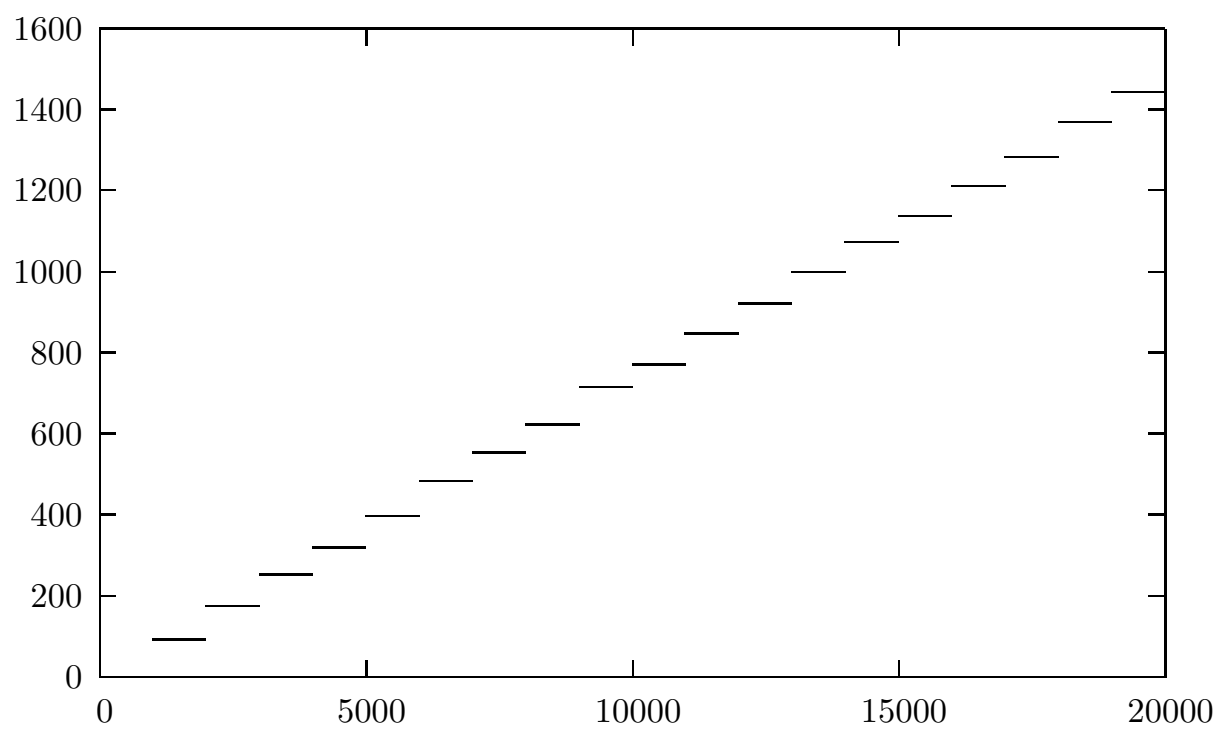

FiguRE 2. The graph of $V$ 
In Table $\mathrm{C}, L\left(m_{1}, m_{2}\right), H\left(m_{1}, m_{2}\right)$ and $R\left(m_{1}, m_{2}\right)$ are numbers defined by $L\left(m_{1}, m_{2}\right):=$ the maximum value of the length of the shortest period of the expansion of $\left(\left\langle\tau_{m}\right\rangle,\left\langle\tau_{m}^{2}\right\rangle\right)$ by our algorithm for $m_{1} \leq m \leq m_{2}$, where $\tau_{m}$ is a maximal root of $x^{3}-m x+1$,

$$
\begin{aligned}
H\left(m_{1}, m_{2}\right) & :=\max _{m_{1} \leq m \leq m_{2}, 0 \leq n<\infty} \operatorname{dh}_{\mathrm{AJPA}}\left(n ;\left\langle\tau_{m}\right\rangle,\left\langle\tau_{m}^{2}\right\rangle\right), \\
R\left(m_{1}, m_{2}\right) & :=\max _{m_{1} \leq m \leq m_{2}, 0 \leq n<\infty} \operatorname{rdh}_{\mathrm{AJPA}}\left(n ;\left\langle\tau_{m}\right\rangle,\left\langle\tau_{m}^{2}\right\rangle\right),
\end{aligned}
$$

which are well defined by the periodicity.

In Table $\mathrm{D}, L\left(m_{1}, m_{2}\right), H\left(m_{1}, m_{2}\right)$ and $R\left(m_{1}, m_{2}\right)$ are numbers defined by $L\left(m_{1}, m_{2}\right):=$ the maximum value of the length of the shortest period of the expansion of $\left(\langle\sqrt[4]{m}\rangle,\left\langle\sqrt[4]{m^{2}}\right\rangle,\left\langle\sqrt[4]{m^{3}}\right\rangle\right)$ by our algorithm for $m_{1} \leq m \leq m_{2}$ with $\sqrt{m} \notin \mathbb{Q}$,

$$
\begin{aligned}
& H\left(m_{1}, m_{2}\right):=\max _{m_{1} \leq m \leq m_{2}, \sqrt{m} \notin \mathbb{Q}, 0 \leq n<\infty} \operatorname{dh}_{\mathrm{AJPA}}\left(n ;\langle\sqrt[3]{m}\rangle,\left\langle\sqrt[3]{m^{2}}\right\rangle,\left\langle\sqrt[3]{m^{3}}\right\rangle\right), \\
& R\left(m_{1}, m_{2}\right):=\max _{m_{1} \leq m \leq m_{2}, \sqrt{m} \notin \mathbb{Q}, 0 \leq n<\infty} \operatorname{rdh}_{\mathrm{AJPA}}\left(n ;\langle\sqrt[3]{m}\rangle,\left\langle\sqrt[3]{m^{2}}\right\rangle,\left\langle\sqrt[3]{m^{3}}\right\rangle\right)
\end{aligned}
$$

which are well defined by the periodicity.

\section{TABLE C}

\begin{tabular}{l|r|r|r}
\hline $\begin{array}{l}\text { range of } m \\
m_{1} \leq m \leq m_{2}\end{array}$ & $L\left(m_{1}, m_{2}\right)$ & $H\left(m_{1}, m_{2}\right)$ & $R\left(m_{1}, m_{2}\right)$ \\
\hline $3 \leq m \leq 200$ & 866 & 6 & 2 \\
\hline $201 \leq m \leq 400$ & 3312 & 7 & $7 / 3$ \\
\hline $401 \leq m \leq 600$ & 5378 & 7 & $7 / 3$ \\
\hline $601 \leq m \leq 800$ & 10578 & 9 & $9 / 4$ \\
\hline $801 \leq m \leq 1000$ & 11808 & 8 & 2 \\
\hline $1001 \leq m \leq 1200$ & 19264 & 8 & 2 \\
\hline $1201 \leq m \leq 1400$ & 17254 & 8 & 2 \\
\hline $1401 \leq m \leq 1600$ & 25792 & 9 & $9 / 4$ \\
\hline $1601 \leq m \leq 1800$ & 33562 & 10 & $5 / 2$ \\
\hline $1801 \leq m \leq 2000$ & 36476 & 9 & $9 / 4$ \\
\hline $2001 \leq m \leq 2200$ & 23274 & 9 & $9 / 4$ \\
\hline $2201 \leq m \leq 2400$ & 38938 & 9 & $9 / 4$ \\
\hline $2401 \leq m \leq 2600$ & 54046 & 10 & $5 / 2$ \\
\hline $2601 \leq m \leq 2800$ & 57246 & 9 & $9 / 4$ \\
\hline $2801 \leq m \leq 3000$ & 51964 & 9 & $9 / 4$ \\
\hline $3001 \leq m \leq 3200$ & 57036 & 9 & $9 / 4$ \\
\hline $3201 \leq m \leq 3400$ & 92332 & 9 & $9 / 4$ \\
\hline $3401 \leq m \leq 3600$ & 55698 & 10 & $5 / 2$ \\
\hline $3601 \leq m \leq 3800$ & 96972 & 9 & $9 / 4$ \\
\hline $3801 \leq m \leq 4000$ & 86784 & 10 & $5 / 2$ \\
\hline $4001 \leq m \leq 4200$ & 94188 & 10 & $5 / 2$ \\
\hline $4201 \leq m \leq 4400$ & 116912 & 10 & $5 / 2$ \\
\hline $4401 \leq m \leq 4600$ & 109288 & 10 & $5 / 2$ \\
\hline $4601 \leq m \leq 4800$ & 113792 & 10 & $5 / 2$ \\
\hline $4801 \leq m \leq 5000$ & 109426 & 10 & $5 / 2$ \\
\hline & & &
\end{tabular}


TABLE D

\begin{tabular}{l|r|r|r}
\hline $\begin{array}{l}\text { range of } m \\
m_{1} \leq m \leq m_{2}\end{array}$ & $L\left(m_{1}, m_{2}\right)$ & $H\left(m_{1}, m_{2}\right)$ & $R\left(m_{1}, m_{2}\right)$ \\
\hline $2 \leq m \leq 200$ & 4194 & 13 & $13 / 5$ \\
\hline $201 \leq m \leq 400$ & 8994 & 12 & 2 \\
\hline $401 \leq m \leq 600$ & 9730 & 11 & $11 / 6$ \\
\hline $601 \leq m \leq 800$ & 18894 & 13 & $13 / 7$ \\
\hline $801 \leq m \leq 1000$ & 14172 & 13 & $13 / 7$ \\
\hline $1001 \leq m \leq 1200$ & 27876 & 14 & 2 \\
\hline $1201 \leq m \leq 1400$ & 34308 & 13 & $12 / 7$ \\
\hline $1401 \leq m \leq 1600$ & 16452 & 13 & $13 / 8$ \\
\hline $1601 \leq m \leq 1800$ & 32870 & 14 & $7 / 4$ \\
\hline $1801 \leq m \leq 2000$ & 44244 & 13 & $13 / 8$ \\
\hline $2001 \leq m \leq 2200$ & 48732 & 13 & $13 / 8$ \\
\hline $2201 \leq m \leq 2400$ & 58974 & 14 & $7 / 4$ \\
\hline $2401 \leq m \leq 2600$ & 62706 & 13 & $13 / 8$ \\
\hline $2601 \leq m \leq 2800$ & 41678 & 13 & $13 / 8$ \\
\hline $2801 \leq m \leq 3000$ & 45066 & 14 & $7 / 4$ \\
\hline $3001 \leq m \leq 3200$ & 41382 & 13 & $13 / 8$ \\
\hline $3201 \leq m \leq 3400$ & 112670 & 15 & $15 / 8$ \\
\hline $3401 \leq m \leq 3600$ & 121296 & 15 & $15 / 8$ \\
\hline $3601 \leq m \leq 3800$ & 58782 & 15 & $5 / 3$ \\
\hline $3801 \leq m \leq 4000$ & 60890 & 13 & $13 / 9$ \\
\hline $4001 \leq m \leq 4200$ & 92190 & 14 & $14 / 9$ \\
\hline $4201 \leq m \leq 4400$ & 43882 & 14 & $14 / 9$ \\
\hline $4401 \leq m \leq 4600$ & 94542 & 16 & $16 / 9$ \\
\hline $4601 \leq m \leq 4800$ & 79714 & 13 & $13 / 9$ \\
\hline $4801 \leq m \leq 5000$ & 51786 & 15 & $5 / 3$ \\
\hline & & &
\end{tabular}

\section{REFERENCES}

1. L. Bernstein, The Jacobi-Perron algorithm-Its theory and application. Lecture Notes in Mathematics, Vol. 207, Springer-Verlag, Berlin, New York (1971). MR0285478 (44:2696)

2. L. Elsner, H. Hasse, Numerische Ergebnisse zum Jacobischen Kettenbruchalgorithmus in reinkubischen Zahlkörpern. (German) Math. Nachr. 34 (1967), 95-97. MR0219508 (36:2589)

3. T. Fujita, Sh. Ito, M. Keane, and M. Ohtsuki, On almost everywhere exponential convergence of modified Jacobi-Perron algorithm: a corrected proof, Ergodic theory an dynamical systems. 16 (1996), 1345-1352. MR.1424403 (98a:11105)

4. S. Ito, J. Fujii, H. Higashino and S. Yasutomi, On simultaneous approximation to $\left(\alpha, \alpha^{2}\right)$ with $\alpha^{3}+k \alpha-1=0$, J. Number Theory 1 (2003), 255-283. MR1968452 (2004a:11063)

5. S. Ito and S. Yasutomi, On simultaneous approximation to certain periodic points related to modified Jacobi-Perron algorithm, to appear in Advanced Studies in Pure Mathematics.

6. GiNaC web site: http://www.ginac.de/.

7. D.N. Lehmer, On Jacobi's Extension of the Continued Fraction Algorithm, Proc Natl Acad Sci U S A. 1918 December; 4(12): 360-364.

8. C.D. Olds, Continued fractions. Random House, New York 1963. MR0146146 (26:3672)

9. O. Perron, Grundlagen für eine Theorie des Jacobischen Kettenbruchalgorithmus, Math. Ann. 64 (1907), 1-76. MR:1511422 
10. E.V. Podsypanin, A generalization of continued fraction algorithm that is related to the Viggo Brun algorithm (Russian), Studies in Number Theory (LOMI), 4, Zap. Naucn. Sem. Leningrad. Otdel. Mat. Inst. Steklov, 67 (1977), 184-194. MR0457337 (56:15545)

11. F. Schweiger, Multidimensional continued fractions. Oxford Science Publications. Oxford University Press, Oxford, 2000. MR2121855(2005i:11090)

12. J. Tamura, A new approach to higher dimensional continued fractions, preprint.

13. J. Tamura, A class of transcendental numbers having explicit $g$-adic and Jacobi-Perron expansions of arbitrary dimension. Acta Arith. 71 (1995), no. 4, 301-329. MR.1339134 (96g:11083)

3-3-7-307 Azamino Aoba-Ku, Yokohama, 225-0011 Japan

E-mail address: jtamura@tsuda.ac.jp

General Education, Suzuka National College of Technology, Shiroko Suzuka Mie 510-0294, JAPAN

E-mail address: yasutomi@genl.suzuka-ct.ac.jp 